Proceedings of the 5th Workshop on Quantum Chaos and Localisation Phenomena, Warsaw, Poland, May 20-22, 2011

\title{
Space-Time Resolved Experiments for Water Waves
}

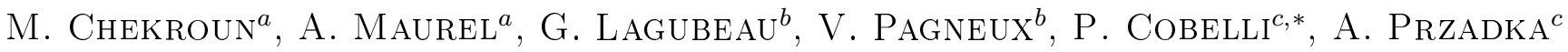 \\ AND P. PETITJEANS ${ }^{c}$
}

${ }^{a}$ Institut Langevin/LOA and UMR CNRS 7587, ESPCI, UPD Univ. Paris 7

10 rue Vauquelin, 75231 Paris Cedex 05, France

${ }^{b}$ Laboratoire d'Acoustique de l'Université du Maine and UMR CNRS 6613

Av. Olivier Messiaen, 72085 Le Mans, France

${ }^{c}$ Physique et Mécanique des Milieux Hétérogènes, UMR CNRS 7636, ESPCI, UPMC Univ. Paris 6

UPD Univ. Paris 7, 10 rue Vauquelin, 75005 Paris, France

\begin{abstract}
An overview of recent works on water wave propagation using a full time-space resolved method is given. The experimental method allows us to precisely measure the surface elevation field with spatial and temporal resolutions given by the pixel size and frequency acquisition of a high speed camera. Two typical problems are regarded: (i) the propagation of water waves through surface piercing obstacles with trapped modes or directional emission, a problem of interest notably for its practical applications to the protection of floating structures and to the canalization of the water wave energy, (ii) a study of water wave turbulence is also reported, exhibiting the interest to measure the joint space-time power spectrum to study which hypothesis of weak turbulence theory survives in laboratory experiments.
\end{abstract}

PACS: 47.35.Bb, 04.30.Nk

\section{Introduction}

The very rich physics of waves is often difficult to measure due to the complexity in space and time of the phenomena encountered. Among the different domains (electromagnetism, quantum mechanics, acoustics, ...) where experiments on waves are carried out, the ability to measure the dynamics in space and time is rare because of very short time scale or small space scale. One kind of waves seems to be a good candidate to succeed in space-time resolved measurements: these are gravity-capillary waves at the surface of water. Indeed, water waves present a direct analogy with linear scalar waves in other contexts of physics. Assuming the flow is irrotational and incompressible, the velocity potential $\phi(x, y, z, t)$ satisfies the Laplace equation $\Delta \phi=0$, with appropriate boundary conditions. On the bottom $z=-H_{0}$, the Neumann boundary condition to account for nonpenetrable bottom

$$
\partial_{z} \phi\left(x, y,-H_{0}\right)=0 .
$$

The classical kinematic condition accounts for the condition that the vertical velocity must match the motion of the free surface and dynamic boundary conditions on the free surface (basically, the Bernoulli equation expressed at the free surface). Linearizing for small deformation of the surface, it yields in the frequency regime with $e^{-i \omega t}$ dependence,

* Present address: Departamento de Fisica, FCEN, UBA and IFIBA, CONICET, Ciudad Universitaria, 1428 Buenos Aires, Argentina.

$$
\begin{aligned}
& \partial_{z} \phi(x, y, 0)=-\mathrm{i} \omega h(x, y), \\
& \mathrm{i} \omega \phi(x, y, 0)=g h(x, y),
\end{aligned}
$$

with $g$ the gravity constant and $H(x, y)$ the local free surface height and $H=H_{0}+h$, where $H_{0}$ is the height of the water at rest. The explicit dependence in the $z$ direction is

$$
\phi(x, y, z)=\cosh k\left(z+H_{0}\right) \varphi(x, y),
$$

with $k$ satisfying the dispersion relation (for gravity waves)

$$
\omega^{2}=g k \tanh k H_{0} .
$$

The new two-dimensional potential $\varphi(x, y)$ is simply proportional to the wave field $h(x, y)$ and the Laplace equation becomes the 2D Helmholtz equation

$$
\left(\Delta+k^{2}\right) h(x, y)=0 .
$$

When capillary effects are taken into account, it is sufficient to replace $g k$ in Eq. (4) by $g k+\gamma k^{3} / \rho$, where $\gamma$ is the surface tension constant.

Besides, beyond the ability of water waves to experience similar behaviors to waves in other domains of physics, gravity-capillary waves have their own interest. The nonlinearities of water waves produce spectacular phenomena. Tsunami or freak waves are examples that have received unceasing attention [1]. Also, the low wave speeds of water waves make easy the appearance of trans/ supersonic wave regimes. For this reason, water waves have been used as the analog of wave experiencing strong interactions, notably in quantum mechanics. As examples, the water waves propagating on a moving counter flow is studied as the analog of quantum fields propa- 
gating around black holes [2]; also, water waves interacting with a bathtub vortex is studied as the analog with a beam of particles interacting with a magnetic field, a problem known in quantum mechanics as the Aharonov-Bohm effects [3].

Recently, we proposed the application of the Fourier transform profilometry (FTP) [4] to study different cases of complex wave phenomena with water waves [5-9]. The FTP is a technique based on the analysis of the deformation of fringes projected onto the surface whose shape measurement is wanted. This yields a measurement of the deformation of the surface, in our case the wave field. Coupled to a high speed camera, it is able to produce a "movie" of the measured wave field with a very good resolution both in space and time.

We report in this paper experiments of gravity waves in different contexts. In the first part, we focus on the problem of linear water wave propagating through obstacles that are surface piercing scatterers. This configuration is used to show the existence of trapped modes localized in the vicinity of the scatterers and also to test the ability to obtain directional emission with a cluster of periodically distributed scatterers. In the second part, we present a statistical analysis of non-linear random waves. There, the space-time resolved measurements obtained owing to our technique allows us to study the joint space-time power spectrum.

\section{Experimental setup, FTP measurements}

The experimental setup consists of a tank filled with water in which different contexts of wave propagation are produced (Fig. 1). The main originality of our setup is the full space-time resolved method of measurement of the surface elevation. Beyond the usual visualizations or measurements at one point, we developed an optical method of measurement of the free surface. The method has been described in detail in Refs. [4-6]. It is based on the analysis of the deformation of fringes projected on the free surface of a light diffusive liquid. The surface depth information is encoded into a deformed fringe pattern recorded by the acquisition sensor, allowing it to be measured by comparison to the original (undeformed) grating image. It is therefore the phase shift between the reference and deformed images which contains all the information of the deformed surface.

We proposed recently significant improvements of the technique (1) by developing a filter free demodulation method [10] and (2) by analyzing carefully the properties of the diffusive liquid to find suspensions that allow to make high diffusivity with, when needed, the same properties as clean water, namely weak attenuation of the gravity-capillary waves [11]. As it is now, the method permits us to measure the 2D field with: (1) the spatial and temporal resolutions of the camera (in our case, a v9 phantom high speed camera with up to more than $1000 \mathrm{fps}$ ) and (2) with an accuracy in the measured height given by the periodicity of the projected fringes (in our experiments, up to $10 \mu \mathrm{m}$ accuracy has been obtained [9]).

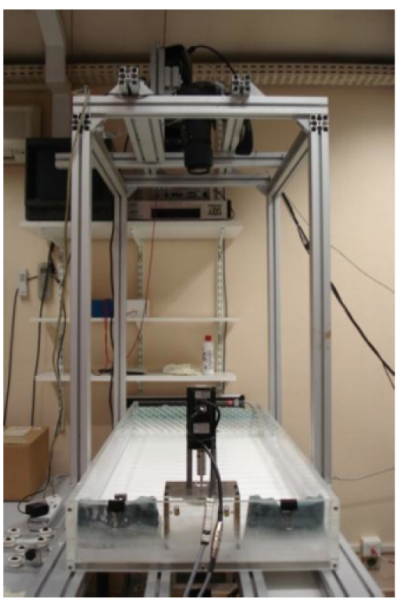

Fig. 1. Experimental setup. The main tank is filled with a light diffusive liquid. The method for free surface measurement needs a projector for the fringe projection and an acquisition sensor (camera) as on the top of the photography.

\section{Propagation of water waves through obstacles}

The problem of the propagation of water waves through an ensemble of scatterers has many applications in naval and coastal engineering. Among these, water wave resonances, as can be observed near floating structures has received much attention due to its practical application. Also of interest is the possibility to capture the energy of the ocean waves. This problem has been recently revisited in the framework of the design of metamaterials coming from the community of electromagnetism within the general question of whether or not we are able to control and to canalize water waves. We present in this section experimental studies of these two problems owing to our quantitative measurements of the water wave fields. The aim in studying experimentally such configurations is to test how robust are the theoretical or numerical predictions, based on the 2D Helmholtz equation with idealized boundary condition (as the Neumann boundary conditions on the wall) and idealized flow (irrotational, incompressible). As will be seen, the case of a unique surface piercing cylinder (Sect. 3.1) appears to nicely follow these approximations. To the opposite, the collective effects appearing when several cylinders are considered may considerably affect the theoretical predictions (Sect. 3.2). Of course, the conclusions that we can make regarding the result of a laboratory experiments are not necessary applicable at the ocean scale. This question is addressed in Sect. 3.2.

\subsection{Wave resonance near an obstacle}

In the framework of the classical theory of linearized water waves in unbounded domains, trapped modes consist in non-propagative localized oscillation modes of finite energy occurring at some well-defined frequency and which, in the absence of dissipation, persist in time even 
in the absence of external forcing. The first theoretical example of such trapping mode in the theory of water waves, due to Stokes [12], corresponds to waves which travel in the long-shore direction over a uniformly sloping beach and decay to zero in the seaward direction. A recent review on edge waves can be found in [13] and a description of edge waves in an oceanographic context in [14].

The fluid around a free surface piercing circular cylinder in a long narrow wave tank can exhibit a local oscillation. In practice, the occurrence of near-trapped modes is of particular relevance to offshore structures based on a large number of piles (such as oil rigs, very large floating structures and breakwaters) as it implies the presence of very large loads on individual elements of the array. Recent progress in the understanding of wave interaction with arrays of offshore structures, resonant effects and their consequences for finite arrays has been discussed by [15].

We characterized the trapped mode and we report here the most relevant conclusion of our work (for details see $[7,8])$. Our experimental setup consists of a water tank with constant water level at rest which is chosen to be fixed at $H_{0}=5 \mathrm{~cm}$ (Fig. 2). The system of interest is placed inside the tank: a waveguide formed by two parallel vertical walls $60 \mathrm{~cm}$ long, a distance $2 d=10 \mathrm{~cm}$ apart, has a free surface piercing vertical circular cylinder of diameter $2 a$ ( $a=1$ to $5 \mathrm{~cm}$ ) located symmetrically between the two walls. Water waves are generated by a wavemaker forming an angle of around $45^{\circ}$ with the waveguide axis $O x$ (so symmetric and antisymmetric modes are generated at the entrance of the waveguide) at a frequency $f \in[2,3] \mathrm{Hz}$. Typical free surface deformation fields $h_{T}(x, y, t)$ near resonance are shown in Fig. 2. The acquisition rate of the camera is synchronized with the wavemaker in order to get 200 acquisitions $h_{T}(x, y, t)$ over two periods of the water wave oscillation. For a perfect fluid, in absence of dissipation, the dispersion relation for the water waves is given by $\omega=g k \tanh k H_{0}$ where $\omega$ is the driving pulsation, $k-$ the wave number and $g=9.81 \mathrm{~m} \mathrm{~s}^{-2}$ (the effects of surface tension are neglected).

In order to obtain quantitative characteristics of the resonance, we proceed as follows: the linear part of the signal is obtained by extracting at each position $(x, y)$, the coefficient $h_{1}(x, y)$ of the Fourier series

$$
h_{T}(x, y, t)=\sum_{n} \operatorname{Re}\left[h_{n}(x, y) \mathrm{e}^{\mathrm{i} n \omega t}\right],
$$

(Re denotes the real part) and we find that the weight of the non-linearities $\left|h_{T}-h_{1}\right| /\left|h_{T}\right|$ is less than $15 \% . h_{1}$ is then separated into an even part $h^{\mathrm{e}}$ and an odd part $h^{\mathrm{o}}$. The even field $h^{\mathrm{e}}$ makes the propagative plane mode to appear, that can be used to get a direct measurement of the wave number $k$. The agreement with the theoretical dispersion relation (not reported here, see [36]) is of about $2 \%$.

The odd part $h^{\circ}$ is then analyzed to quantify the behavior of the trapped mode. If correctly governed by the

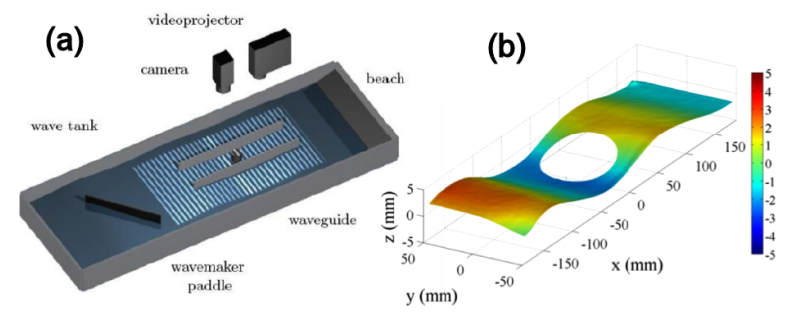

Fig. 2. (a) Experimental setup. Symmetric and antisymmetric modes are generated at the entrance of the waveguide by the wave maker. A free surface piercing circular cylinder of diameter $2 a \in[2,10] \mathrm{cm}$ lies in the center of the waveguide of width $2 d=10 \mathrm{~cm}$. (b) Typical instantaneous field of the surface elevation $h_{T}(x, y, t)$, here for $a / d=0.50, k d=1.32$. The scale of the colorbar is in $\mathrm{mm}$.

Helmholtz equation $\left(\Delta+k^{2}\right) h_{1}(x, y)=0$, with the Neumann boundary condition at the walls, the field can be decomposed onto the modes in the transverse direction $\sin n \pi y / 2 d$ ( $n$ integer). The odd part of the field is composed of evanescent modes only, so that we expect only the resonant mode to be dominant in the far field of the cylinder and the problem reduces to a $1 \mathrm{D}$ problem (in the near field, the higher transverse modes are expected to contribute to the $2 \mathrm{D}$ solution)

$$
h^{\circ}(x, y) \simeq a_{1}(x) \sin \pi y / 2 d,
$$

and for this evanescent mode

$$
\begin{aligned}
& a_{1}(x<0)=A \mathrm{e}^{-\alpha x}+A R \mathrm{e}^{\alpha x}, \\
& a_{1}(x>0)=A T \mathrm{e}^{-\alpha x},
\end{aligned}
$$

with $A$ the amplitude of the incident wave, $\alpha=$ $\sqrt{k^{2}-\pi^{2} /(2 d)^{2}}$ the wave number of the first evanescent mode and $(R, T)$ the reflection and transmission coefficients (Fig. 3). The reflection and transmission coefficients $(R, T)$ are fitted for each frequency, leading to the resonance curves, as exemplified in Fig. 3 for $a / d=0.9$ (two resonances are visible). As is obvious from these curves, the classical Breit-Wigner resonance shape is not pertinent since it is not able to reproduce the asymmetry, a behavior already observed for resonances occurring near the cutoff frequency in [16] and that can be described using a particular resonance shape of the form $T=B /(1-C / \alpha d)$. Typical value of the attenuation part of the wave number used to fit our resonance curves is $1.5 \mathrm{~m}^{-1}$. This value is in good agreement with careful measurements of the attenuation in our experiments [11].

The behavior of the resonance frequency $k_{\mathrm{c}} d$ when changing the size of the cylinder is shown in Fig. 4, that summarizes our main result: we observe two branches. The first corresponds to trapped modes symmetric with respect to $O y$-axis and the second to trapped modes antisymmetric with respect to $O y$-axis. The experimental results are compared to the theoretical predictions of Ref. [17] for the first branch and with the theoretical predictions of Ref. [18] for the second branch. This sec- 

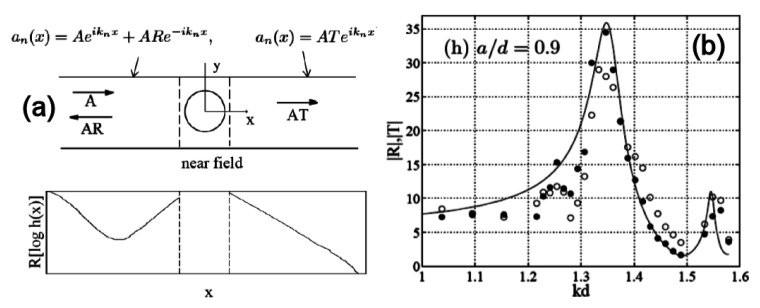

Fig. 3. (a) Reduction of the problem to a $1 \mathrm{D}$ problem. The modal component $h_{1}(x)$ of the first antisymmetric mode (evanescent, $k_{1}=\mathrm{i} \alpha$ ) in the far field allows to get the reflexion $R$ and transmission $T$ coefficients, Eq. (8) (bottom curve is a typical example of the experimental $h_{1}(x)$ behavior in a log-lin representation). (b) Example of the resulting resonance curve as a function of the frequency for $a / d=0.9$, two resonances $k_{\mathrm{c}}$.

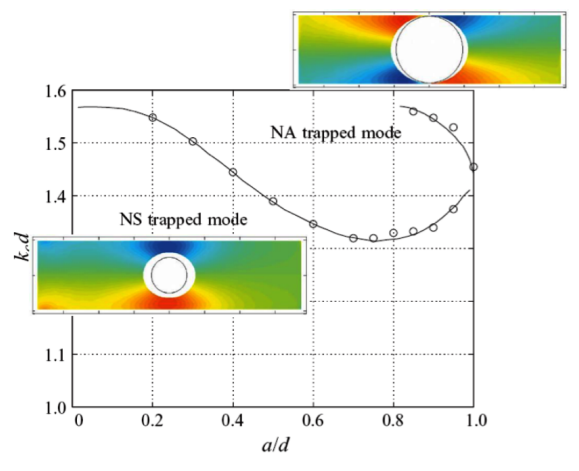

Fig. 4. Resonance frequency $k_{\mathrm{c}} d$ as a function of the aspect ratio $a / d$. Open symbols are our experimental measurements, plane lines are the theoretical predictions by $[17,18]$.

ond branch is predicted to exist for $a / d>0.81$ and it is experimentally confirmed here. Globally, an excellent agreement is observed with the theoretical predictions for both branches. It is worth noting that this is the case even for the largest values of $a / d$, for which the effect of a meniscus in the small region between the cylinder and the waveguide walls might be important. As will be seen in the next section, the dynamics of the contact line at the boundaries are not always negligible.

\subsection{On the possibility of enhanced and directional} water wave emission by a periodic lattice of cylinders

Photonic and phononic crystals have attracted considerable attention due to the existence of frequency band gaps, and the particular behavior of waves that are propagating in corresponding periodical structures. For application to water waves, interesting phenomena have been reported such as the superlensing and self-collimation phenomena $[19,20]$. In a recent paper, Mei and co-authors [21] proposed the design of a highly directional liquid surface wave source by utilizing the high density of states at the band-edge frequency of a periodic structure. Their numerical study exhibits a high directivity associated to a resonance in the wave intensity transmitted by the lattice in one direction. We report below the experimental study of the proposed configuration. It appears that this effect is not robust in our laboratory scale experiments. The measured patterns of transmitted wave intensity nicely compares with numerical calculations but the agreement needs an unexpected high attenuation to be accounted for in the numerical model. This can be due to the attenuation produced by the dynamics associated with moving contact lines [22], a phenomenon that can be less important at the ocean scale.

The experimental setup is shown in Fig. 5. The lattice used in the experiment is composed of brass cylinders of diameter $2 a=2 \mathrm{~cm}$ forming a square lattice with lattice step $d=3 \mathrm{~cm}$ (filling fraction $\pi a^{2} / d^{2}=0.35$ as considered in [21]). The cylinders are placed (immersed) in a wave tank with a water depth $H_{0}=3.3 \mathrm{~cm}$. The point source is produced by a thin tip controlled by a linear motor. The tip oscillates vertically at the free surface with a sinusoidal frequency $\omega$. Results presented below corresponds to $\omega=38.51 \mathrm{~s}^{-1}$, near the expected resonance at $35 \mathrm{~s}^{-1}$. Similar results have been observed at other frequencies [23].

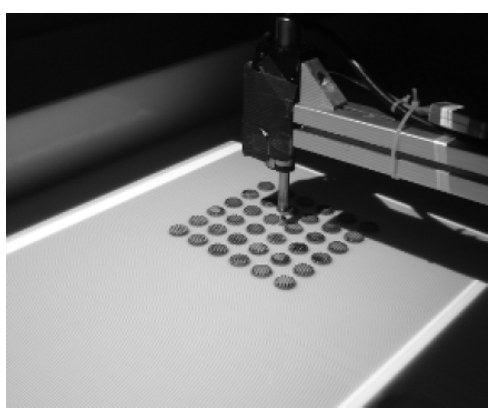

Fig. 5. Experimental setup: a square lattice of $6 \times 6$ cylinders are placed in a wave tank. The point source is produced by a thin tip oscillating vertically at a sinusoidal frequency $\omega$.
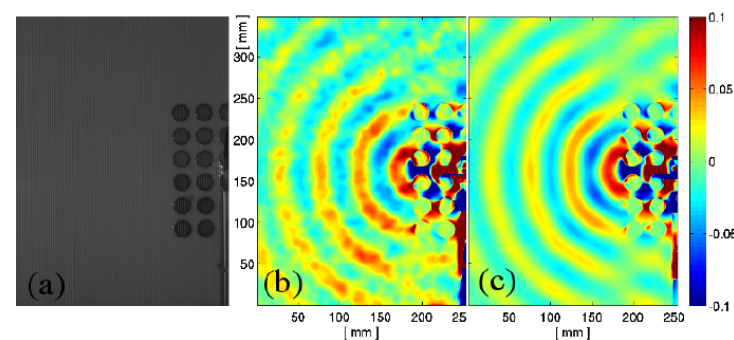

Fig. 6. (a) Direct visualization of the wave field; (b) instantaneous measured field and (c) linear part of the measured field (colorbars in (b) and (c) are in $\mathrm{mm}$ ).

Example of measured instantaneous field $h_{T}(x, y, t)$ using our FTP method is shown in Fig. 6b. After filtering the non-linearities, the complex field $h_{1}(x, y)$ is obtained (Fig. 6c, see Eq. (6)). This allows us to get the wave intensity pattern $\left|h_{1}\right|^{2}$, as illustrated in Fig. 7. The measured water wave intensity can be compared to the pat- 
tern calculated numerically when considering $h_{1}$ satisfies the Helmholtz equation with the Neumann boundary condition on the cylinder boundaries (this is done by using multiple scattering simulations as in [21]). We performed a simulation in which the unique adjustable parameter is the attenuation of the water wave encapsulated in the wave number, assumed to have an imaginary part $\alpha_{\text {num }}$. For $\alpha_{\text {num }}=10 \mathrm{~m}^{-1}$, an excellent agreement is found, both on the $2 \mathrm{D}$ pattern and on the scattering function (wave amplitude $h_{1}(r, \theta) \simeq f(\theta) / \sqrt{r}$ measured at the same distance $r$ from the source with $\theta$ the angular direction). This is illustrated in Fig. 7. As previously said, the attenuation in our experiments has been carefully characterized [11] and we found $\alpha \simeq 3 \mathrm{~m}^{-1}$ for $\omega=38.51 \mathrm{~s}^{-1}$, three times smaller than the one that we have to take in the numerics to reproduce the experimental measurements. The enhancement of the apparent attenuation produced by the dynamics associated with moving contact lines has been studied in detail, see [22] and reference therein. However, in the experiments with a unique cylinder in a waveguide, presented in the previous section, no enhancement of the attenuation has been found. This suggests that the vicinity of the cylinders produces more important effects of the multiple moving contact lines and this leads to the increase of the attenuation. However, the agreement found between numerics and experiments shows that this phenomenon can be simply encapsulated in an extra attenuation, the behavior of the water wave being otherwise very well described by the Helmholtz equation, as considered in numerical calculations.

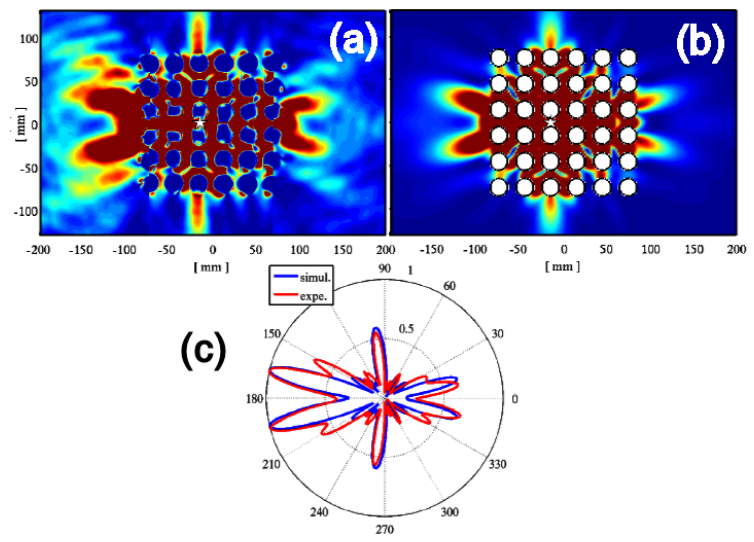

Fig. 7. Patterns of water wave intensity as observed (a) experimentally and (b) in the numerics considering an attenuation $\alpha_{\text {num }}=10 \mathrm{~m}^{-2}$. (c) Comparison of the scattering functions experimentally measured and obtained numerically.

Finally, Fig. 8 shows the wave intensity pattern and the scattering function at the same frequency but neglecting the attenuation $\left(\alpha_{\text {num }}=0\right)$. The directivity that is observed in that case corresponds to the one described in [21]. It appears clearly by comparison with Fig. 7 that the directivity is lost because of the attenuation.

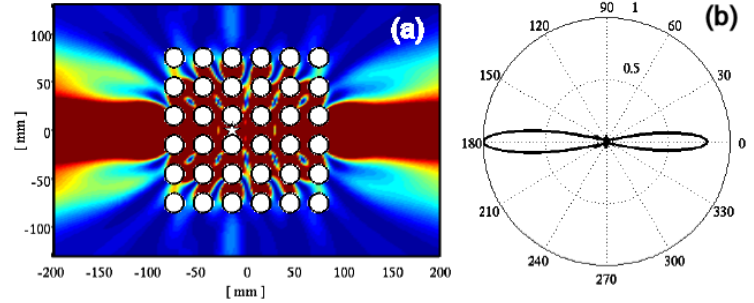

Fig. 8. Directivity expected from numerical calculations (MST, as used in [21]) without attenuation $\alpha_{\text {num }}=0$. (a) Intensity pattern and (b) scattering function for $\omega=38.51 \mathrm{~s}^{-1}$. The position of the source is indicated by a white star.

\section{Wave turbulence}

Wave turbulence (WT) theory predicts that non-linear random waves are able to experience an energy cascade through different scales, similar to the famous Kolmogorov cascade in classical hydrodynamics. In the context of gravity wave [24, 25], the power spectrum is expected to follow:

$$
\left|\eta_{\omega}\right|^{2} \propto P^{1 / 3} \omega^{-4}, \quad\left|\eta_{k}\right|^{2} \propto P^{1 / 3} k^{-5 / 2},
$$

in the inertial range ( $P$ is the injected energy) and this law seems to reasonably fit the spectra measured for ocean waves in many cases $[26,27]$. To get deeper insights into the non-linear behavior of gravity waves, well controlled laboratory experiments have been developed in the recent years [28-31]. However, they have been unable to reproduce the spectrum at the oceanic scale, and a question has emerged on whether or not the conditions for the applicability of WT theory can be in place in such finite systems. Indeed, this theory assumes weak nonlinearities, low attenuation and small finite size effects and meeting these requirements is particularly difficult in a laboratory tank $[32,33]$.

We report here results in a laboratory experiment showing that it is possible to recover the spectrum as predicted within WT theory. This is done using a low frequency forcing bandwidth (as narrow as possible). Indeed, as suggested in [33], this should enlarge the inertial range in the gravity regime. In the presented experiments, the frequency band width has the maximum frequency $\omega_{\mathrm{m}}=9.44 \mathrm{~s}^{-1}$, for a transition between gravity and capillary waves at $\omega_{\mathrm{c}}=85 \mathrm{~s}^{-1}\left(k_{\mathrm{c}} \equiv \sqrt{\rho g / \gamma}=\right.$ $\left.369 \mathrm{~m}^{-1}\right)$. The water waves are generated by two wave makers in a $(177 \times 61) \mathrm{cm}^{2}$ tank filled with water with depth at rest $H_{0}=5 \mathrm{~cm}$. The wave maker motions are controlled by a random signal within a broadband frequency range $[0,9.44] \mathrm{s}^{-1}$ with maximum amplitude $A=30 \mathrm{~mm}$. Also, in these experiments, the sensitivity of the FTP measurement is improved by treating the phase shifts between two successive images (rather than the phase shift with a reference to unperturbed free surface), resulting in the measurement of the velocity of the surface elevation $\dot{\eta}(\boldsymbol{r}, t)$. A typical velocity measured field is shown in Fig. 9. 


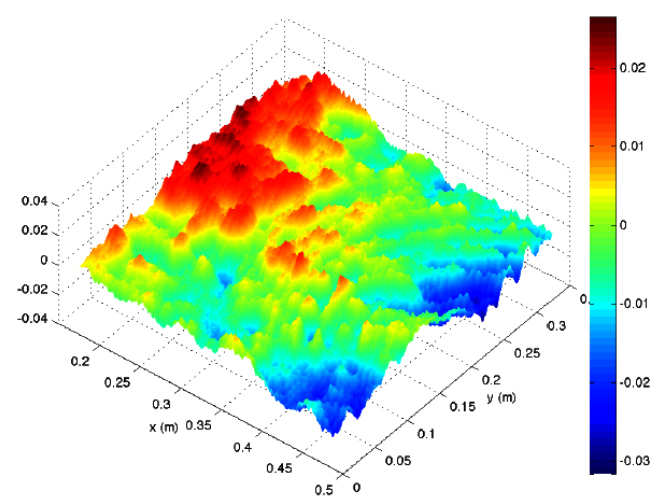

Fig. 9. Typical instantaneous velocity field $\dot{\eta}(\boldsymbol{r}, t)$ (color scale are in $\mathrm{m} / \mathrm{s}$ ) for $A=28 \mathrm{~mm}$.

Our space-time measurement allows to experimentally measure the joint space-time power spectrum

$$
\left|\eta_{\boldsymbol{k}, \omega}\right|^{2} \equiv \int \mathrm{d} \boldsymbol{k} \mathrm{d} \omega\left\langle\eta\left(\boldsymbol{r}^{\prime}, t^{\prime}\right) \eta\left(\boldsymbol{r}+\boldsymbol{r}^{\prime}, t+t^{\prime}\right)\right\rangle \mathrm{e}^{\mathrm{i}(\omega t+\boldsymbol{k} \cdot \boldsymbol{r})},
$$

a quantity that shows how the non-linear interactions spread the wave energy in the 3D $(\boldsymbol{k}, \omega)$-space. This allows us to test the validity of the premise of WT theory that assumes the concentration of the wave energy on the renormalized dispersion relation (which remains close to the linear dispersion relation) and it allows us to evaluate the isotropy of the energy distribution. This is illustrated in Fig. 10. Different features can be seen: on the one hand, the energy indeed spreads isotropically whatever being the considered frequency, which indicates a weak dependence on the tank shape. On the other hand, the energy remains concentrated on the dispersion relation manifold. These are indications that the conditions of applicability of WT theory are satisfied in our experiments. Indeed, it has been observed numerically [33] and experimentally $[34,35]$ that non-linear mode, also called bound waves, can exist. These modes correspond to strong non-linearities with frequencies $n \omega$. They appear near the injection scale and their existence may affect the behavior of the power spectrum.

Finally, the most significant feature is shown in Fig. 11: a forcing independent inertial range is observed with a scaling law in $\omega$ in agreement with the ZF predictions $\left|\eta_{\omega}\right|^{2} \propto \omega^{-4}$. The spectra in the $k$-space are shown in the inset, where the ZF prediction $\left|\dot{\eta}_{k}\right|^{2} \propto k^{-3 / 2}$ are displayed for comparison.

Other experiments (not reported here) have been performed using a higher $\omega_{\mathrm{m}}=25.13 \mathrm{~s}^{-1}$. In that case, bound waves are observed and this is accompanied with the lost of the forcing independent inertial range. Instead, in the regime of gravity wave, the power spectrum is found to have an increasing spectral slope, from -5 to -4 when increasing the forcing amplitude. This behavior is the one usually observed in laboratory experiments [30-32].

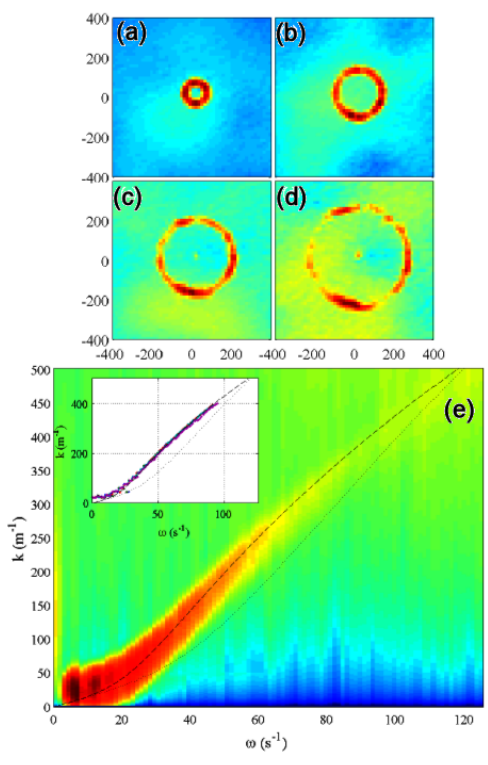

Fig. 10. $\left|\eta_{\boldsymbol{k}, \omega}\right|^{2}$ for (a) $\omega=24 \mathrm{~s}^{-1}$, (b) $\omega=36.4 \mathrm{~s}^{-1}$, (c) $\omega=49 \mathrm{~s}^{-1}$ and (d) $\omega=62 \mathrm{~s}^{-1}(A=3 \mathrm{~mm})$, illustrating the isotropy in the $\left(k_{x}, k_{y}\right)$-space. (e) Typical spectrum $\left|\eta_{k, \omega}\right|^{2}$ at forcing amplitude $A=20 \mathrm{~mm}$. The inset shows the collected dispersion relations for $A=1$, $6,12,20$, and $28 \mathrm{~mm}$. The dashed line shows the linear dispersion relation and the dotted line shows the dispersion relation of the first bound waves.

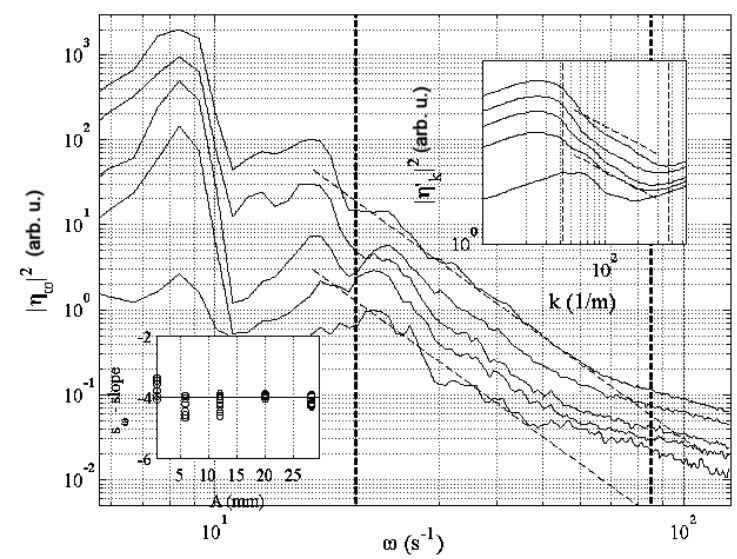

Fig. 11. Spectra $\left|\eta_{\omega}\right|^{2}$ for several forcing amplitude (between $A=1$ and $30 \mathrm{~mm}$ ). Vertical dotted lines at $30 \mathrm{~s}^{-1}$ and at $\omega_{\mathrm{c}}=85 \mathrm{~s}^{-1}$ delimitate the inertial range of the gravity regime. Top inset: corresponding spectra $\left|\dot{\eta}_{k}\right|^{2}$. Bottom inset: spectral exponent as a function of $A$ (multiple symbols correspond to multiple fits in a moving window within the inertial range).

\section{Concluding remarks}

We have shown that space-time resolved measurements of complex wave phenomena can be obtained by using the Fourier transform profilometry for water waves.

When the interest is in the characterization of linear waves in the frequency regime, our method permits to 
filter the signal at the driving frequency. We exemplified this by the study of the wave scattering by the Neumann type scatterers: we characterized precisely the trapped modes around a surface piercing cylinder in a waveguide. Also, we have shown that the directional emission theoretically displayed for a source in a cluster of periodically arranged cylinders is not robust with respect to the attenuation of water in mesoscale laboratory experiments.

The study of wave turbulence corresponds to a case where the nonlinear interactions govern the dynamics of the wave field. In that case, the full space-time characterization is of particular importance. For wave turbulence, it permits to have access to the $(k, \omega)$-space that is the natural space in turbulence theory. We showed that our method is very efficient: the resolution was sufficient to calculate the experimental joint space-time spectrum. A statistical analysis of this spectrum showed a good agreement with the cascade scenario of wave turbulence theory.

\section{Acknowledgments}

The authors thank the Agence Nationale de la Recherche for its funding under grant No. Tourbillonde ANR-08-BLAN-0108-02.

\section{References}

[1] A. Slunyaev, C. Kharif, E. Pelinovsky, Rogue Waves in the Ocean, Springer, Berlin 2009.

[2] S. Weinfurtner, E.W. Tedford, M.C.J. Penrice, W.G. Unruh, G.A. Lawrence, Phys. Rev. Lett. 106, 021302 (2011).

[3] M.V. Berry, R.G. Chambers, M.D. Large, C. Upstill, J.C. Walmsley, Eur. J. Phys. 1, 154 (1980).

[4] M. Takeda, K. Mutoh, Appl. Opt. 22, 3977 (1983).

[5] A. Maurel, P. Cobelli, V. Pagneux, P. Petitjeans, Appl. Opt. 48, 380 (2009).

[6] P. Cobelli, A. Maurel, V. Pagneux, P. Petitjeans, $E x$ periments Fluids 46, 1037 (2009).

[7] P. Cobelli, V. Pagneux, A. Maurel, P. Petitjeans, J. Fluid Mech. 666, 445 (2011).

[8] P. Cobelli, V. Pagneux, A. Maurel, P. Petitjeans, Europ. Phys. Lett. 88, 20006 (2009).

[9] G. Lagubeau, M. Fontelos, C. Josserand, A. Maurel, V. Pagneux, P. Petitjeans, Phys. Rev. Lett. 105, 184503 (2010).

[10] G. Lagubeau, A. Maurel, V. Pagneux, P. Petitijeans, EMD algorithm applied to fringe projection profilometry, preprint.

[11] A. Przadka, B. Cabane, V. Pagneux, A. Maurel, P. Petitjeans, Exp. in Fluids 52, 519 (2011).

[12] G.G. Stokes, Report on Recent Researches in Hydrodynamics, in: Report to 16th Meeting Brit. Assoc. Adv. Sci., Southampton, 1846, p. 120.
[13] R.S. Johnson, Philos. Trans. R. Soc. A 365, 2359 (2007).

[14] P.H. LeBlond, L.A. Mysak, Waves in the Ocean, Elsevier, Amsterdam 1978.

[15] M. McIver, R. Porter, J. Fluid Mech. 456, 277 (2002).

[16] E. Granot, Phys. Rev. B 65, 233101 (2002).

[17] M. Callan, C.M. Linton, D.V. Evans, J. Fluid Mech. 229, 51 (1991).

[18] D.V. Evans, R. Porter, J. Eng. Math. 35, 149 (1999).

[19] M. Torres, J.P. Adrados, F.R. Montero de Espinosa, Nature 398, 114 (1999).

[20] X. Hu, Y. Shen, X. Liu, R. Fu, J. Zi, X. Jiang, S. Feng, Phys. Rev. E 68, 037301 (2003).

[21] J. Mei, C. Qiu, J. Shi, Z. Liu, Wave Motion 47, 131 (2010).

[22] L.M. Hocking, J. Fluid Mech. 179, 253 (1987).

[23] M. Chekroun, A. Maurel, V. Pagneux, P. Petitjeans, Hyperfocalisation of water waves through an array of surface piercing cylinders, preprint.

[24] V.E. Zakharov, V.S. Lvov, G. Falkovich, Kolmogorov Spectra of Turbulence, Springer-Verlag, Berlin 1992.

[25] A.C. Newell, B. Rumpf, Ann. Rev. Fluid. Mech. 1, 60 (2011).

[26] V.E. Zakharov, AGU Geophysical Monograph, Miami 2005, http://math.arizona.edu/ zakharov/1Articles/Cascades.pdf.

[27] The WISE Group, Progr. Oceanogr. 75, 603 (2007).

[28] W.B. Wright, R. Budakian, S.J. Putterman, Phys. Rev. Lett. 76, 4528 (1996).

[29] G.V. Kolmakov, A.A. Levchenko, M.Yu. Brazhnikov, L.P. Mezhov-Deglin, A.N. Silchenko, P.V.E. McClintock, Phys. Rev. Lett. 93, 074501 (2004).

[30] E. Falcon, C. Laroche, S. Fauve, Phys. Rev. Lett. 98, 094503 (2007); E. Falcon, Discrete Contin. Dyn. Syst. B 13, 81940 (2010).

[31] P. Denissenko, S. Lukaschuk, S. Nazarenko, Phys. Rev. Lett. 99, 014501 (2007).

[32] S. Lukaschuk, S. Nazarenko, S. McLelland, P. Denissenko, Phys. Rev. Lett. 103, 044501 (2009); S. Nazarenko, S. Lukaschuk, S. McLelland, P. Denissenko, J. Fluid Mech. 642, 395 (2010).

[33] Y.V. Lvov, S. Nazarenko, B. Pokorni, Physica D 218 , 24 (2006).

[34] E. Herbert, N. Mordant, E. Falcon, Phys. Rev. Lett. 105, 144502 (2010).

[35] W.J. Plant, J. Geophys. Res. 109, C10002 (2004); H.E. Krogstad, K. Trulsen, Ocean Dynamics 60, 973 (2010).

[36] P. Cobelli, A. Przadka, P. Petitjeans, G. Lagubeau, V. Pagneux, A. Maurel, Phys. Rev. Lett. 107, 214503 (2011). 